\title{
PRÉ-CONCENTRAÇÃO BASEADA NA COPRECIPITAÇÃO USANDO CROMATO DE PRATA COMO CARREADOR PARA DETERMINAÇÃO DE COBRE POR FAAS
}

\author{
Ivero P. Sáa, Luana N. Santos ${ }^{a}$, Erik G. P. da Silva ${ }^{a}$, Daniel de C. Lima ${ }^{a}$ e Fábio Alan C. Amorima,*,(D)

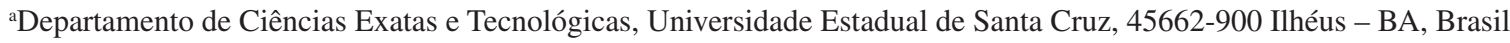

Recebido em 09/05/2018; aceito em 02/10/2018; publicado na web em 29/11/2018

\begin{abstract}
PRECONCENTRATION BASED ON COPRECIPITATION USING SILVER CHROMATE AS A CARRIER FOR COPPER DETERMINATION BY FAAS. In the present paper, a preconcentration procedure based in the coprecipitation of copper by silver dichromate $\left(\mathrm{Ag}_{2} \mathrm{CrO}_{4}\right)$ and consecutive determination by flame atomic absorption spectrometry (FAAS) was proposed. The experimental factors were optimized from application of the Doehlert matrix and the conditions set: chromate concentration $5.8 \times 10^{-4} \mathrm{~mol} \mathrm{~L}^{-1}$, silver concentration $1.1 \times 10^{-3} \mathrm{~mol} \mathrm{~L}^{-1}, \mathrm{pH} 7.3$ and disperser concentration $\mathrm{HNO}_{3} 0.38 \mathrm{~mol} \mathrm{~L}^{-1}$. Under these conditions, analytical parameters of the procedure were obtained, with the quantification limits of $0.76 \mu \mathrm{g} \mathrm{L}^{-1}$, precision (based in the relative standard deviation, RSD \%) with values lower than $2.7 \%$, and preconcentration factor of 28 . The accuracy of the procedure was given by addition/recovery test in water samples groundwater and mineral, and by comparison with results obtained by ICP-MS. The procedure developed presented be simple, sensitive and low cost, thus can be applied in the preconcentration and determination of copper in several water samples.
\end{abstract}

Keywords: copper; coprecipitation; silver chromate; Doehlert matrix; FAAS.

\section{INTRODUÇÃO}

O cobre é um elemento de importância considerável nos sistemas ambientais e na fisiologia animal. ${ }^{1}$ A essencialidade do cobre é firmemente estabelecida na dieta nutricional humana, e este apresenta um papel fundamental na síntese de diversas enzimas responsáveis pela produção de proteínas. ${ }^{1,2}$ Apesar de sua relevância, a toxicidade do $\mathrm{Cu}^{2+}$ desempenha um papel importante no desenvolvimento da doença de Alzheimer, mostrando que até pequenas quantidades deste analito em água potável causa a rápida perda da cognição. ${ }^{1-5}$ Por isso, é de extrema necessidade o monitoramento desse analito em amostras ambientais, alimentos e de água.

Técnicas espectroanalíticas convencionais como a espectrometria de absorção atômica com chama (FAAS) tem sido eficiente na detecção de metais traços em vários tipos de amostras devido a sua alta seletividade, rapidez, fácil manuseio e baixo custo. No entanto, FAAS apresenta algumas limitações como a impossibilidade de determinar analitos em baixas concentrações e/ou alta influência de sais dissolvidos em amostras de águas, causando interferência no sinal dos analitos. ${ }^{6-9}$ Para solucionar essas limitações, vários métodos de separação/pré-concentração têm sido empregados, como, por exemplo, a extração em fase sólida, ${ }^{10}$ extração em ponto nuvem, ${ }^{11}$ extração por solventes, ${ }^{12}$ flotação, ${ }^{13}$ adsorção, ${ }^{14}$ coprecpitação, ${ }^{15} \mathrm{e}$ membrana filtrante. ${ }^{16,17}$

A coprecipitação é um importante método de separação/pré-concentração para metais traços devido a sua simplicidade, baixo consumo de solvente e alto fator de pré-concentração. Além disso, diversos analitos podem ser separados e pré-concentrados em uma única etapa da matriz utilizando diferentes coprecipitantes, orgânicos ou inorgânicos. ${ }^{18-21}$ Hidróxidos metálicos como érbio, ${ }^{22}$ cério, ${ }^{23}$ túlio, ${ }^{7}$ gadolínio, ${ }^{24}$ zircônio $^{15}$ têm sido empregados como coprecipitantes inorgânicos. Coprecipitantes orgânicos que tendem a formar quelatos neutros com espécies metálicas como 8-hidroxiquinolina, ditiocarbamato e ácido violúrico têm sido usados na pré-concentração de metais traços em várias matrizes. ${ }^{25,26} \mathrm{~A}$ coprecipitação envolve a

*e-mail: facamorim@uesc.br incorporação de traços de impurezas de uma fase líquida solúvel para dentro do precipitado. A habilidade dos precipitados de capturar impurezas pode ser utilizada para concentrar elementos traço. ${ }^{27}$ Os mecanismos de coprecipitação incluem a adsorção superficial, a troca iônica, precipitação superficial e a oclusão. ${ }^{28}$

$\mathrm{O}$ cromato de prata $\left(\mathrm{Ag}_{2} \mathrm{CrO}_{4}\right)$, composto bastante conhecido e empregado na química analítica clássica com indicador para quantificação de íons cloreto pelo método de Mohr. O coprecipitante cromato de prata $\left(\mathrm{Ag}_{2} \mathrm{CrO}_{4}\right)$ é praticamente insolúvel em água, com $\mathrm{Kps}=1,12 \times 10^{-12} \mathrm{e}$ apresenta um interessante equilíbrio químico dependente do $\mathrm{pH}$ com a espécie dicromato, que é solúvel. ${ }^{29} \mathrm{O}$ mecanismo de coprecipitação predominante é baseado no fenômeno de adsorção superficial, em que após a precipitação do cromato de prata forma-se a primeira camada de adsorção na qual são retidos os analitos, os quais são contrabalanceados com a camada de adsorção secundária. Ainda que seja um composto popular e comumente utilizado como indicador em métodos titulométricos de precipitação, a aplicação do $\mathrm{Ag}_{2} \mathrm{CrO}_{4}$ como carreador para separação e pré-concentração por coprecipitação não foi avaliada.

Diante do exposto, o objetivo deste trabalho foi desenvolver um método de pré-concentração com o emprego do carreador cromato de prata para coprecipitação de quantidades traço de $\mathrm{Cu}^{2+}$ em amostras de águas para determinação por FAAS.

\section{PARTE EXPERIMENTAL}

\section{Instrumentos}

Um espectrômetro de absorção atômica com chama, modelo SpectrAA 240FS (VARIAN, Mulgrave, Austrália) equipado com chama ar-acetileno foi utilizado para detecção do analito. As condições instrumentais foram: comprimento de onda do cobre $324,8 \mathrm{~nm}$, corrente da lâmpada $10 \mathrm{~mA}$, largura da fenda $0,5 \mathrm{~nm}$, altura do queimador 13,5, mm, vazão de acetileno $2,00 \mathrm{~L} \mathrm{~min}^{-1}$ e vazão de ar 13,5 $\mathrm{L} \mathrm{min}^{-1}$.

Um espectrômetro de massas com plasma indutivamente acoplado (ICP-MS) modelo NexION 300X (Perkin-Elmer, Shelton, $\mathrm{CT}$ ) equipado com a tecnologia de célula universal (Universal Cell 
Technology ${ }^{\mathrm{TM}}$ - UCT) foi utilizado para determinações diretas de cobre $\left({ }^{63} \mathrm{Cu}\right)$ nas amostras. As condições intrumentais foram: potência RF $1600 \mathrm{~W}$, vazão de gás no plasma $18 \mathrm{~L} \mathrm{~min}^{-1}$, vazão de gás auxiliar $1,2 \mathrm{~L} \mathrm{~min}^{-1}$, vazão de gás no nebulizador $1,01 \mathrm{~L} \mathrm{~min}^{-1}$.

As medidas dos $\mathrm{pH}$ foram realizadas em pHmetro HANNA modelo $\mathrm{pH}$ 21, uma centrífuga Solab modelo L-700 foi utilizada para acelerar o processo de decantação do precipitado, uma balança analítica GEHAKA foi utilizada para medir as massas de reagentes e amostras. Uma centrífuga Eppendorf modelo 5804 foi utilizada para decantação do precipitado.

\section{Reagentes e soluções}

Para o preparo de todas as soluções foi utilizado água desionizada obtida por osmose reversa (18,2 $\mathrm{M} \Omega \mathrm{cm}$, GEHAKA modelo OS10 LX). Todos reagentes utilizados foram de grau analítico. Toda vidraria utilizada foi descontaminada em solução $\mathrm{HNO}_{3} 10 \%$ (v/v) por 24 horas e lavada duas vezes com água desionizada.

As soluções padrão de $\mathrm{Cu}$ (II) foram preparadas a partir da diluição do padrão estoque $1000 \mathrm{mg} \mathrm{L}^{-1}$ (Specsol). Soluções de cromato de potássio $\left(\mathrm{K}_{2} \mathrm{CrO}_{4}\right.$, Merck) e nitrato de prata $\left(\mathrm{AgNO}_{3}\right.$, Vetec $)$ foram preparadas a $0,055 \mathrm{~mol} \mathrm{~L}^{-1}$. Tris (Sigma-Aldrich) $0,1 \mathrm{~mol} \mathrm{~L}^{-1}$ foi preparado e o pH ajustado de acordo ao experimento. Ácido nítrico 65 $\%$ m/m (Merck) foi utilizado para preparação das soluções de ácido nítrico utilizadas para solubilização do precipitado.

\section{Método de pré-concentração}

O método de coprecipitação do cromato de prata foi otimizado em soluções padrões. Em tubos de ensaio contendo $10 \mathrm{~mL}$ de solução-amostra contendo $\mathrm{Cu}^{2+}$ a $20 \mu \mathrm{g} \mathrm{L}^{-1}$ foram adicionados $5,8 \times 10^{-4} \mathrm{~mol} \mathrm{~L}^{-1}$ de cromato e $1,1 \times 10^{-3} \mathrm{~mol} \mathrm{~L}^{-1}$ de prata. $\mathrm{O}$ pH da solução foi ajustado para 7,3 utilizando solução tampão tris $0,1 \mathrm{~mol} \mathrm{~L}^{-1}$. O tubo foi agitado manualmente por alguns segundos e em seguida foi centrifugado em $1780 \mathrm{G}$ durante $10 \mathrm{~min}$. O sobrenadante foi removido e o precipitado permaneceu aderido ao tubo, e este foi solubilizado com $200 \mu \mathrm{L}$ de $\mathrm{HNO}_{3} 0,38 \mathrm{~mol} \mathrm{~L}{ }^{-1}$. O cobre foi determinado por espectrometria de absorção atômica com chama, e como resultado analítico foi utilizado absorvância relativa a área do pico do analito em função do tempo (10 s) para $200 \mu \mathrm{L}$ de amostra pré-concentrada.

\section{Estratégia de otimização}

O procedimento de coprecipitação foi otimizado através da análise dos quatro fatores: (1) concentração de cromato; (2) concentração de prata; (3) pH; (4) concentração do dispersor $\mathrm{HNO}_{3}$. Os níveis foram determinados a partir da revisão de trabalhos publicados e experimentos preliminares. ${ }^{30-34}$ A matriz Doehlert foi empregada para a otimização dos fatores, sendo os experimentos realizados em triplicata. Para análise dos dados foi utilizado o programa STATISTICA tendo nível de confiança fixo em $95 \%$ e a Tabela 1 mostra os fatores e níveis estudados neste procedimento.

\section{Aplicação em amostras reais}

As amostras de água mineral engarrafada foram coletadas na cidade de Ilhéus, BA, Brasil e as amostras de água subterrânea coletadas em vários pontos no bairro do Banco da Vitória e em bairros da região norte da cidade de Ilhéus-Ba, Brasil. As amostras foram acondicionadas em frascos de polipropileno e mantidas refrigeradas em aproximadamente $4{ }^{\circ} \mathrm{C}$ e transportadas até o laboratório. No laboratório, antes da aplicação do procedimento de pré-concentração, as amostras foram filtradas utilizando membrana de acetato de celulose com poros de $0,45 \mu \mathrm{m}$, acidificadas com $\mathrm{HNO}_{3}$ a pH 1 e armazenadas sob refrigeração entre 2 e $4{ }^{\circ} \mathrm{C}$. $\mathrm{O}$ pH das amostras foi ajustado para pH 7,3 com solução tampão Tris. Posteriormente, o procedimento de pré-concentração descrito anteriormente foi aplicado às amostras, sempre em triplicata.

\section{RESULTADOS E DISCUSSÃO}

\section{Otimização das condições experimentais}

O emprego de ferramentas quimiométricas é uma interessante opção na otimização de processos de pré-concentração. A maior vantagem na aplicação dessas ferramentas está na análise da interação das variáveis estudadas. Dentre as ferramentas utilizadas têm-se a matriz Doehlert, o planejamento Box-Behnker e o planejamento de composto central que têm sido amplamente descritos na literatura. ${ }^{35-39}$

Para execução da otimização do procedimento de coprecipitação utilizou-se a matriz Doehlert, e foram necessários 24 experimentos expresso em uma matriz com 4 fatores, sendo 21 experimentos e mais 3 experimentos no ponto central, a qual está demonstrada na Tabela 1 .

Os resultados foram analisados de acordo ao percentual de recuperação, e estes foram calculados a partir do fator de pré-concentração teórico do procedimento.

\section{Validação do modelo estatístico e experimental}

A avaliação da qualidade do modelo matemático é necessária para a obtenção de dados confiáveis, ou seja, se este é capaz de descrever o comportamento dos valores experimentais satisfatoriamente. A qualidade do modelo matemático pode ser testada por meio da Análise de Variância (ANOVA).

Os modelos matemáticos, linear e quadrático foram avaliados a fim de obter a melhor descrição da região experimental. Segundo Pimentel e Neto, ${ }^{40}$ para avaliar a qualidade do modelo, deve-se verificar o valor $\mathrm{F}$ da falta de ajuste. Se o modelo matemático estiver ajustado aos dados experimentais, a média quadrática da falta de ajuste $\left(\mathrm{MQ}_{\mathrm{faj}}\right)$ deve refletir apenas os erros aleatórios inerentes ao sistema. Além disso, a média quadrática do erro puro $\left(\mathrm{MQ}_{\mathrm{ep}}\right)$ também deve ser uma boa estimativa desses erros e presume-se que estes dois valores não são estatisticamente diferentes. Se a razão entre a $\mathrm{MQ}_{\mathrm{faj}} / \mathrm{MQ}_{\mathrm{ep}}$ for inferior ao valor de $\mathrm{F}$ tabelado, o ajuste do modelo é considerado satisfatório.

Com ANOVA apresentada na Tabela 2, observou-se que a falta de ajuste para o modelo quadrático não é significativa, pois o valor da razão da $\mathrm{MQ}_{\mathrm{faj}} / \mathrm{MQ}_{\mathrm{ep}} \mathrm{e}(8,01)$ inferior ao valor de $\mathrm{F}_{\text {tabelado }}(8,78)$ para 10 e 3 graus de liberdade, respectivamente. Assim, o modelo quadrático está bem ajustado aos dados obtidos apresentando menores resíduos e desta forma boa capacidade de previsão.

Além do teste da falta de ajuste, o modelo quadrático foi avaliado também pela análise do gráfico dos valores preditos em função dos valores observados, apresentado na Figura 1(a). A concordância entre os valores preditos pelo modelo e os valores experimentais $\left(\mathrm{R}^{2}=0,93\right)$ confirma que o modelo quadrático está bem ajustado aos dados experimentais obtidos no procedimento, com percentagem de variação explicada em torno de $93 \%$.

O modelo quadrático também foi avaliado através do gráfico de valores preditos em função dos resíduos. Assim como a falta de ajuste apresenta importância na avaliação do modelo, é necessário que avalie os resíduos deixados pelo modelo. Para um modelo bem ajustado, esses resíduos não devem apresentar nenhum indício de anormalidade.

Um modelo que deixa resíduos muito grandes ou tendenciosos é inadequado para inferir precisamente sobre o comportamento destes dados no campo experimental em questão. ${ }^{40,41}$ A Figura 1(b) apresenta o gráfico de resíduos e, pode-se observar que os valores dos resíduos 
Tabela 1. Variáveis experimentais com o intervalo estudado, e matriz de Doehlert com os valores reais, valores codificados, e recuperações obtidas em cada experimento

\begin{tabular}{|c|c|c|c|c|c|c|}
\hline \multicolumn{4}{|c|}{ Variáveis } & \multicolumn{2}{|c|}{ Intervalo estudado } & $\mathrm{N}^{\circ}$ de níveis estudados \\
\hline \multicolumn{4}{|c|}{$\mathrm{pH}$} & \multicolumn{2}{|c|}{$4,0-8,8$} & 7 \\
\hline \multicolumn{4}{|c|}{ Concentração de cromato $\left(\left[\mathrm{CrO}_{4}{ }^{2-}\right], \mathrm{mol} \mathrm{L}^{-1}\right)$} & \multicolumn{2}{|c|}{$2,75 \times 10^{-4}-8,25 \times 10^{-4}$} & 7 \\
\hline \multicolumn{4}{|c|}{ Concentração de prata $\left(\left[\mathrm{Ag}^{+}\right], \mathrm{mol} \mathrm{L}^{-1}\right)$} & \multicolumn{2}{|c|}{$5,5 \times 10^{-4}-1,65 \times 10^{-3}$} & 5 \\
\hline \multicolumn{4}{|c|}{ Concentração do dispersor $\left(\left[\mathrm{HNO}_{3}\right], \mathrm{mol} \mathrm{L}^{-1}\right)$} & \multicolumn{2}{|c|}{$0,1-0,5$} & 3 \\
\hline $\operatorname{Exp}^{\mathrm{a}}$ & {$\left[\mathrm{CrO}_{4}{ }^{2-}\right]$} & {$\left[\mathrm{Ag}^{+}\right]$} & $\mathrm{pH}$ & {$\left[\mathrm{HNO}_{3}\right]$} & Absorvância & \% Recuperação \\
\hline 1 & $5,50(0)^{b}$ & $1,10(0)$ & $6,4(0)$ & $0,3(0)$ & 0,1763 & 65,4 \\
\hline 2 & $8,25(1)$ & $1,10(0)$ & $6,4(0)$ & $0,3(0)$ & 0,1857 & 69,0 \\
\hline 3 & $6,87(0,5)$ & $1,65(0,866)$ & $6,4(0)$ & $0,3(0)$ & 0,2002 & 74,5 \\
\hline 4 & $6,87(0,5)$ & $1,28(0,289)$ & $8,8(0,817)$ & $0,3(0)$ & 0,1714 & 63,5 \\
\hline 5 & $6,87(0,5)$ & $1,28(0,289)$ & $7,2(0,204)$ & $0,5(0,791)$ & 0,1951 & 72,6 \\
\hline 6 & $2,75(-1)$ & $1,10(0)$ & $6,4(0)$ & $0,3(0)$ & 0,1897 & 70,5 \\
\hline 7 & $4,21(-0,5)$ & $0,550(-0,866)$ & $6,4(0)$ & $0,3(0)$ & 0,1977 & 73,6 \\
\hline 8 & $4,21(-0,5)$ & $0,918(-0,289)$ & $4,0(-0,817)$ & $0,3(0)$ & 0,1414 & 52,4 \\
\hline 9 & $4,21(-0,5)$ & $0,918(-0,289)$ & $5,6(-0,204)$ & $0,1(-0,791)$ & 0,1561 & 57,9 \\
\hline 10 & $6,87(0,5)$ & $0,550(-0,866)$ & $6,4(0)$ & $0,3(0)$ & 0,1647 & 60,9 \\
\hline 11 & $6,87(0,5)$ & $0,918(-0,289)$ & $4,0(-0,817)$ & $0,3(0)$ & 0,1399 & 51,5 \\
\hline 12 & $6,87(0,5)$ & $0,918(-0,289)$ & $5,6(-0,204)$ & $0,1(0,791)$ & 0,1573 & 58,3 \\
\hline 13 & $4,21(-0,5)$ & $1,65(0,866)$ & $6,4(0)$ & $0,3(0)$ & 0,1724 & 63,9 \\
\hline 14 & $5,50(0)$ & $1,47(0,577)$ & $4,0(-0,817)$ & $0,3(0)$ & 0,1315 & 48,3 \\
\hline 15 & $5,50(0)$ & $1,47(0,577)$ & $5,6(-0,204)$ & $0,1(-0,791)$ & 0,1523 & 56,5 \\
\hline 16 & $4,21(-0,5)$ & $1,28(0,289)$ & $8,8(0,817)$ & $0,3(0)$ & 0,1766 & 65,5 \\
\hline 17 & $5,50(0)$ & $0,735(-0,577)$ & $8,8(0,817)$ & $0,3(0)$ & 0,1718 & 63,7 \\
\hline 18 & $5,50(0)$ & $1,1(0)$ & $8,0(0,613)$ & $0,1(-0,791)$ & 0,1678 & 62,2 \\
\hline 19 & $4,21(-0,5)$ & $1,28(0,289)$ & $7,2(0,204)$ & $0,5(0,791)$ & 0,1724 & 63,9 \\
\hline 20 & $5,50(0)$ & $0,735(-0,577)$ & $7,2(0,204)$ & $0,5(0,791)$ & 0,1995 & 74,2 \\
\hline 21 & $5,50(0)$ & $1,1(0)$ & $4,8(-0,613)$ & $0,5(0,791)$ & 0,1433 & 52,8 \\
\hline 22 & $5,50(0)$ & $1,1(0)$ & $6,4(0)$ & $0,3(0)$ & 0,1870 & 69,5 \\
\hline 23 & $5,50(0)$ & $1,1(0)$ & $6,4(0)$ & $0,3(0)$ & 0,1924 & 71,6 \\
\hline 24 & $5,50(0)$ & $1,1(0)$ & $6,4(0)$ & $0,3(0)$ & 0,1870 & 69,5 \\
\hline
\end{tabular}

${ }^{\mathrm{a}}$ Experimentos; ${ }^{\mathrm{b}}$ Valores codificados entre parênteses.

Tabela 2. ANOVA usando o percentual de recuperação para o modelo, com um nível de confiança de $95 \%$

\begin{tabular}{|c|c|c|c|c|c|}
\hline Fator & SQ & $\mathrm{gl}$ & MQ & $\mathrm{F}_{\text {calculado }}$ & p-valor \\
\hline$\left[\mathrm{CrO}_{4}{ }^{2-}\right](\mathrm{Q})$ & 0,633 & 1 & 0,6330 & 0,09435 & 0,778810 \\
\hline$\left[\mathrm{Ag}^{+}\right](\mathrm{Q})$ & 2,565 & 1 & 2,5651 & 0,38233 & 0,580130 \\
\hline $\mathrm{pH}(\mathrm{Q})$ & 456,440 & 1 & 456,4397 & 68,03437 & 0,003731 \\
\hline$\left[\mathrm{HNO}_{3}\right](\mathrm{Q})$ & 96,241 & 1 & 96,2408 & 14,34512 & 0,032278 \\
\hline$\left[\mathrm{CrO}_{4}{ }^{2-}\right](\mathrm{L}) \mathrm{X}\left[\mathrm{Ag}^{+}\right](\mathrm{L})$ & 28,124 & 1 & 28,1238 & 4,19198 & 0,133092 \\
\hline$\left[\mathrm{CrO}_{4}{ }^{2-}\right](\mathrm{L}) \mathrm{X} \mathrm{pH}(\mathrm{L})$ & 81,466 & 1 & 81,4658 & 12,14284 & 0,039921 \\
\hline$\left[\mathrm{CrO}_{4}{ }^{2-}\right](\mathrm{L}) \mathrm{X}\left[\mathrm{HNO}_{3}\right](\mathrm{L})$ & 29,785 & 1 & 29,7855 & 4,43966 & 0,125719 \\
\hline$\left[\mathrm{Ag}^{+}\right](\mathrm{L}) \mathrm{X} \mathrm{pH}(\mathrm{L})$ & 4,045 & 1 & 4,0446 & 0,60286 & 0,494085 \\
\hline$\left[\mathrm{Ag}^{+}\right](\mathrm{L}) \mathrm{X}\left[\mathrm{HNO}_{3}\right](\mathrm{L})$ & 12,021 & 1 & 12,0208 & 1,79175 & 0,273114 \\
\hline $\mathrm{pH}(\mathrm{L}) \mathrm{X}\left[\mathrm{HNO}_{3}\right](\mathrm{L})$ & 10,706 & 1 & 10,7061 & 1,59579 & 0,295751 \\
\hline Falta de ajuste & 537,903 & 10 & 53,7903 & 8,01768 & 0,056661 \\
\hline Erro puro & 20,127 & 3 & 6,7090 & & \\
\hline Total SQ & 1372,936 & 23 & & & \\
\hline
\end{tabular}

SQ-soma quadrática; gl-grau de liberdade; MQ-média quadrática. ANOVA usando o percentual de recuperação para o modelo, com um nível de confiança de 95\% 

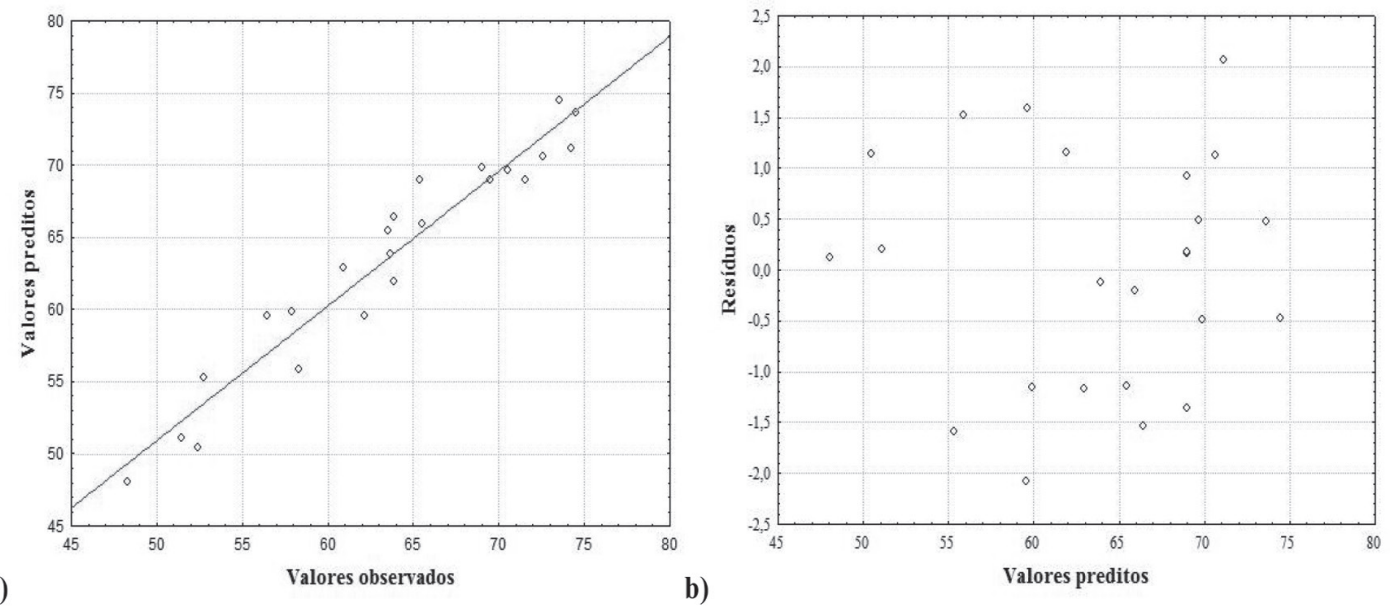

Figura 1. (a) Gráfico de valores preditos em função dos valores observados e (b) gráfico de resíduos em função dos valores preditos para o modelo quadrático usando o percentual de recuperação

são de magnitude na faixa de $\pm 2,2 \%$ de recuperação e se distribuem aleatoriamente mostrando mais uma vez que o modelo quadrático está bem ajustado.

A determinação das condições ótimas para os dados modelados pode ser realizada a partir da equação da regressão apresentada na equação 1. É possível calcular as coordenadas do ponto crítico, ou seja, estabelece as condições em que os fatores estudados fornecem a melhor resposta, através das primeiras derivadas desta função matemática que descreve a superfície de resposta, em relação a cada variável e igualando-as a zero. ${ }^{41}$ Atraves desses cálculos, foram obtidos os valores críticos para cada variável estudada, como sendo: $\left[\mathrm{CrO}_{4}{ }^{2-}\right]=5,8 \times 10^{-4},\left[\mathrm{Ag}^{+}\right]=1,1 \times 10^{-3},\left[\mathrm{HNO}_{3}\right]=0,38 \mathrm{e} \mathrm{pH}=7,3$.

$\mathrm{R} \%=68,0028_{ \pm 1,2534}-0,2054_{ \pm 1,2093}\left(\left[\mathrm{CrO}_{4}^{2-}\right]\right)+0,4357_{ \pm 1,2617}$ $\left(\left[\mathrm{CrO}_{4}{ }^{2-}\right]\right)^{2}-3,3651_{ \pm 1,1949}\left(\left[\mathrm{Ag}^{+}\right]\right)-0,9453_{ \pm 1,6823}\left(\left[\mathrm{Ag}^{+}\right]\right)^{2}+$ $8,6950_{ \pm 1,1658}(\mathrm{pH})-11,4236_{ \pm 1,4104}(\mathrm{pH})^{2}+2,5042_{ \pm 1,1536}\left(\left[\mathrm{HNO}_{3}\right]\right)$ $-4,6346_{ \pm 1,2708}\left(\left[\mathrm{HNO}_{3}\right]\right)^{2}+8,7186_{ \pm 1,9426}\left(\left[\mathrm{CrO}_{4}^{2-}\right]\right) *\left(\left[\mathrm{Ag}^{+}\right]\right)-$ $3,2704_{ \pm 2,0451}\left(\left[\mathrm{Ag}^{+}\right]\right) *(\mathrm{pH})+1,3035_{ \pm 2,0915}\left(\left[\mathrm{CrO}_{4}{ }^{2-}\right]\right) *\left(\left[\mathrm{HNO}_{3}\right]\right)+$ $1,7558_{ \pm 2,3726}\left(\left[\mathrm{CrO}_{4}^{2-}\right]\right) *(\mathrm{pH})-3,2712_{ \pm 2,4237}\left(\left[\mathrm{CrO}_{4}^{2-}\right]\right) *\left(\left[\mathrm{HNO}_{3}\right]\right)+$ $2,7165_{ \pm 2,1992}(\mathrm{pH}) *\left(\left[\mathrm{HNO}_{3}\right]\right)$

A inspeção visual das superfícies de resposta é outra forma de extrair informações do modelo referente as condições que melhor beneficiam a recuperação de cobre para esse procedimento. ${ }^{42}$ Entretanto, só é possível projetar duas variáveis em cada projeção gráfica e o terceiro eixo é dedicado a resposta analítica. Nas superfícies de resposta obtidas, a região entre os eixos onde a cor vermelha é mais intensa denota o máximo da resposta para os fatores projetados. Três, das seis, superfícies de resposta são apresentadas na Figura 2, e todas elas apresentam pontos de máximo coerentes com os pontos críticos obtidos.

$\mathrm{O}$ pH influencia diretamente no equilíbrio químico de formação do coprecipitante, cromato de prata. Em pH mais ácido o equilíbrio é deslocado para formação do íon dicromato, equação 2 , ao qual é solúvel e isto implica diretamente na recuperação de cobre. ${ }^{43} \mathrm{Em}$ pH elevado a concentração de $\mathrm{OH}^{-}$interfere no equilíbrio favorecendo a formação do hidróxido de prata (equação 3). A precipitação da prata como hidróxido só ocorre em $\mathrm{pH}$ superior a 10 e por este motivo a faixa de trabalho não excedeu ao $\mathrm{pH} 9 .{ }^{43} \mathrm{~A}$ condição que maximiza a precipitação de $\mathrm{Ag}_{2} \mathrm{CrO}_{4}$ para a extração de cobre em relação ao $\mathrm{pH}$ foi determinado pelos valores críticos do modelo e da superfície de resposta.

$$
\begin{aligned}
& 2 \mathrm{CrO}_{4}^{2-}{ }_{(\mathrm{aq})}+2 \mathrm{H}^{+}{ }_{(\mathrm{aq})} \leftrightharpoons \mathrm{Cr}_{2} \mathrm{O}_{7}{ }^{2-}{ }_{\text {(aq) }}+\mathrm{H}_{2} \mathrm{O}_{(\mathrm{l})} \\
& \mathrm{Ag}^{+}{ }_{(\mathrm{aq})}+\mathrm{OH}_{(\mathrm{aq})}^{-} \leftrightharpoons \mathrm{AgOH}_{(\mathrm{s})}
\end{aligned}
$$

As concentrações de cromato e de prata são de fundamental importância para o procedimento, pois não há coprecipitação sem o precipitado cromato de prata. A concentração do agente dispersor $\mathrm{HNO}_{3}$ é um importante fator, necessário para solubilização do precipitado liberando assim o cobre para a solução, a adição do ácido nítrico deslocou o equilíbio para a formação do dicromato o que favoreceu a solubilização do precipitado disponibilizando o cobre para a determinação. Assim, as condições críticas obtidas pelos cálculos $\left(\left[\mathrm{CrO}_{4}{ }^{2-}\right]\right.$ $=5,8 \times 10^{-4},\left[\mathrm{Ag}^{+}\right]=1,1 \times 10^{-3},\left[\mathrm{HNO}_{3}\right]=0,38 \mathrm{e} \mathrm{pH}=7,3$ ) corroboram com a inspeção visual dos pontos máximos de resposta, estando todos os valores críticos dentro do domínoi experimental estudado.

\section{Características analíticas}

Sob as condições otimizadas foram estabelecidos os parâmetros analíticos do procedimento e os mesmos foram determinados de acordo com as recomendações da União Internacional de Química Pura e Aplicada (IUPAC) ${ }^{44}$ A curva de calibração foi construída variando a concentração de cobre de 2 até $100 \mu \mathrm{g} \mathrm{L}^{-1}$, com equação para a reta sendo Abs $=0,0028_{ \pm 0,0004}\left[\mathrm{Cu}, \mu \mathrm{g} \mathrm{L}^{-1}\right]-0,0168_{ \pm 0,0075}$ e R $\mathrm{R}^{2}=0,9987$. $\mathrm{O}$ fator de enriquecimento experimental foi calculado utilizando a relação entre os coeficientes angulares das curvas de calibração com e sem pré-concentração, e o valor calculado foi de 28. Considerando a razão entre os volumes da amostra $(10 \mathrm{~mL})$ e da fase rica $(0,2 \mathrm{~mL})$, o fator de enriquecimento teórico é de 50, resultando numa eficiência de transferencia de fases de $56 \%$.

O limite de detecção (LD), como a concentração equivalente a 3 vezes o desvio padrão do branco, obtido por 10 determinações distintas, dividido pela inclinação da curva analítica, foi de $0,23 \mu \mathrm{g} \mathrm{\textrm {L } ^ { - 1 }}$. O limite de quantificação (LQ), como a concentração equivalente a 10 vezes o desvio padrão do branco, obtido por 10 determinações distintas, dividido pela inclinação da curva analítica, foi de $0,77 \mu \mathrm{g} \mathrm{L}^{-1}$.

A precisão do procedimento foi avaliada como desvio padrão relativo (RSD \%) de 10 determinações distinas nas concentrações $5,0 \mu \mathrm{g} \mathrm{L}^{-1}$ e $100 \mu \mathrm{g} \mathrm{L}^{-1}$ e os valores encontrados foram $2,7 \%$ e $1,3 \%$, respectivamente.

\section{Efeito de outros íons}

Um dos problemas que mais compromete uma análise em espectrometria de absorção atômica na detecção de metais traços é a interferência de matriz. Esses experimentos devem ser conduzidos para examinar o efeito de outros íons na coprecipitação, porque alguns desses metais traços estão presentes em amostras reais e pode 

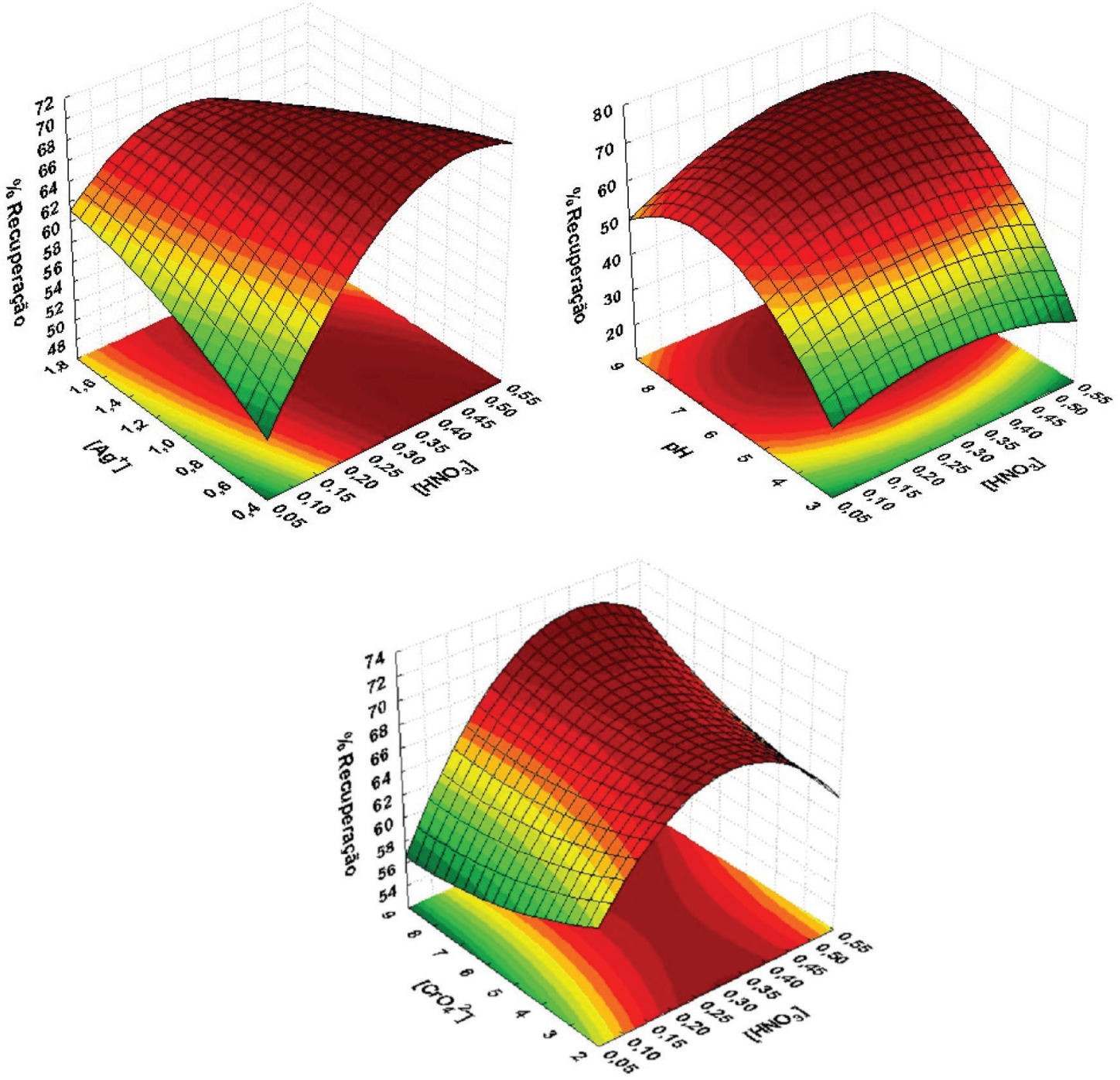

Figura 2. Superfícies de resposta obtidas a partir da matriz. Doehlert para as variáveis

interferir tanto na determinação do analito quanto no procedimento de coprecipitação desenvolvido. Neste estudo, diferentes quantidades de outros íons foram adicionadas a uma solução contendo cobre a $20 \mu \mathrm{g} \mathrm{L}^{-1}$ e a amostra tratada de acordo ao procedimento recomendado. Os resultados obtidos mostraram que as recuperações foram quantitativas para o cobre na presença de outros elementos (Tabela 3). O limite de tolerância foi definido como a concentração de íons que conduz a um erro menor que $5 \%$ em relação a separação e detecção do analito. Elevadas concentrações do íon cloreto (> 30\%o) podem inviabilizar a aplicação do procedimento de coprecipitação, pois na presença deste íon a prata pode precipitar como cloreto de prata e não como cromato de prata. A constante de solubilidade do cromato de prata é $1,12 \times 10^{-12}$ e do cloreto de prata $1,77 \times 10^{-10}$, apesar do $\mathrm{Ag}_{2} \mathrm{CrO}_{4}$ apresentar $\mathrm{Kps}$ menor, o $\mathrm{AgCl}$ é o precipitado formado, o que ocorre devido à solubilidade molar do cromato de prata ser maior que a do cloreto de prata. Considerando esse fator, o presente método não é aplicado a análises de maostra de água salobra ou salgada, isso é, contendo íons cloreto em concentrações maiores que $5 \%$.

\section{Exatidão e aplicação}

A exatidão de um procedimento é determinada pela proximidade do valor medido com o valor real da amostra. A acurácia pode
Tabela 3. Efeito de íons sobre a recuperação de cobre no prodecimento de coprecipitação $(n=3)$

\begin{tabular}{lccc}
\hline Íons & Adicionado como & Proporção & Recuperação (\%) \\
\hline $\mathrm{NO}_{3}{ }^{-}$ & $\mathrm{KNO}_{3}$ & $1: 250$ & 98,5 \\
$\mathrm{PO}_{4}{ }^{3-}$ & $\mathrm{Na}_{3} \mathrm{PO}_{4}$ & $1: 50$ & 95,2 \\
$\mathrm{SO}_{4}^{2-}$ & $\mathrm{Na}_{2} \mathrm{PO}_{4}$ & $1: 50$ & 97,9 \\
$\mathrm{Ca}^{2+}$ & $\mathrm{Ca}\left(\mathrm{NO}_{3}\right)_{2}$ & $1: 50$ & 94,5 \\
$\mathrm{Mg}^{2+}$ & $\mathrm{Mg}\left(\mathrm{NO}_{3}\right)_{2}$ & $1: 50$ & 96,7 \\
$\mathrm{Na}^{+}$ & $\mathrm{NaNO}_{3}$ & $1: 500$ & 93,9 \\
$\mathrm{~K}^{+}$ & $\mathrm{KNO}_{3}$ & $1: 250$ & 95,8 \\
$\mathrm{Ni}^{2+}, \mathrm{Cd}^{2+}, \mathrm{Hg}^{2+}$, & $\mathrm{Nitratos}^{2+}$ & $1: 20$ & 96,3 \\
$\mathrm{Mn}^{2+}, \mathrm{Co}^{2+}$ & & & \\
\hline
\end{tabular}

ser medida de três formas, a primeira delas é a análise da amostra com concentração conhecida e comparação do valor medido com o verdadeiro, vários materiais de referência são utilizados com essa finalidade. A segunda maneira é comparar os resultados do procedimento desenvolvido com os resultados de um método alternativo existente que é conhecido por ser preciso. A terceira abordagem é mais utilizada em estudos de recuperação, e é realizada por adição de um analito em amostras. ${ }^{45}$ 
Para avaliar a exatidão do procedimento, diferentes quantidades de analitos foram adicionadas em amostras de água mineral, água subterrânea. O procedimento de coprecipitação foi aplicado às amostras de águas e os resultados estão apresentados na Tabela 4. Obteve-se uma ótima concordância entre as quantidades de analitos adicionadas e encontradas. Os valores de recuperação para os analitos variaram entre 94 e $98 \%$. Estes valores foram quantitativos e confirmam a exatidão do procedimento proposto, mostrando que o método de coprecipitação apresentado pode ser aplicado à separação e pré-concentração de cobre em amostras de água subterrânea e mineral.

Tabela 4. Recuperação de cobre em amostras de água enriquecidas usando o procedimento de coprecipitação (resultados média \pm desvio padrão, $n=3$ )

\begin{tabular}{lccc}
\hline Amostra & $\begin{array}{c}\text { Adicionada } \\
\left(\mu \mathrm{g} \mathrm{L}^{-1}\right)\end{array}$ & $\begin{array}{c}\text { Encontrada } \\
\left(\mu \mathrm{g} \mathrm{L}^{-1}\right)\end{array}$ & Recuperação \% \\
\hline \multirow{2}{*}{ Água de mineral } & 0 & $4,17 \pm 0,11$ & --- \\
& 5 & $8,97 \pm 0,23$ & 96,0 \\
& 50 & $52,9 \pm 0,9$ & 97,5 \\
\hline \multirow{2}{*}{ Âgua de subter- } & 0 & $4,57 \pm 0,21$ & --- \\
rânea & 5 & $9,48 \pm 0,26$ & 98,2 \\
& 50 & $53,3 \pm 2,13$ & 97,5 \\
\hline
\end{tabular}

Após verificar a exatidão do procedimento proposto, a coprecipitação de cobre foi aplicada para pré-concentração em outras 6 amostras de água subterrânea amostradas em diferentes localidades da região litorânea norte da cidade de Ilhéus, Bahia, Brasil. Além disso, foi realizado uma comparação entre os valores obtidos por análise em FAAS e por análise direta destas mesmas amostras por ICP-MS (Tabela 5).

A avaliação estatística aplicando o teste- $t$ pareado ao nível de confiança de $95 \%$ mostrou não haver diferença estatística entre os valores obtidos pelo procedimento de coprecipitação e aqueles obtidos por análise direta no ICP-MS, considerando que o $t$ calculado é de 0,09 , enquanto que o $t$-crítico foi de 2,78 .

O Conselho Nacional de Meio Ambiente (CONAMA) preconiza que em águas de classe 1, tidas como própria para o consumo humano, os valores máximos permitidos para cobre não devem ser superiores
Tabela 5. Aplicação do método de coprecipitação proposto em amostras águas de subterrâneas (resultados média \pm desvio padrão, $n=3$ )

\begin{tabular}{ccc}
\hline \multirow{2}{*}{ Amostras } & \multicolumn{2}{c}{ Concentração das amostras $\left(\mu \mathrm{g} \mathrm{L}{ }^{-1}\right)$} \\
\cline { 2 - 3 } & FAAS & ICP-MS \\
\hline 1 & $4,57 \pm 0,10$ & $4,38 \pm 0,65$ \\
2 & $4,11 \pm 0,11$ & $4,10 \pm 0,61$ \\
3 & $4,62 \pm 0,09$ & $4,81 \pm 0,72$ \\
4 & $8,11 \pm 0,20$ & $8,42 \pm 1,26$ \\
5 & $1,09 \pm 0,02$ & --- \\
6 & $1,66 \pm 0,04$ & --- \\
\hline
\end{tabular}

a $9 \mu \mathrm{g} \mathrm{L} \mathrm{L}^{-1}$. Nas amostras analisadas todas as concentrações de cobre obtidas estão abaixo do valor máximo permitido pelo CONAMA. ${ }^{46}$

O procedimento de coprecipitação desenvolvido apresentou LQ bem mais baixo que o limíte máximo permitido pela legislação brasileira, consumo de pequeno volume de amostra, e mostrou uma eficiência de extração superior a outros procedimentos descritos na literatura, evidenciado principalmente pelo parâmetro índice de consumo, expresso pela razão entre volume amostra e o fator de enriquecimento, conforme apresentado na Tabela 6.

\section{CONCLUSÃO}

Nesse trabalho foi proposto um procedimento de separação e pré-concentração baseado na coprecipitação de cobre usando como agente carreador o cromato de prata, e esse procedimento foi aplicado nas amostras de água subterrânea e água mineral. Com a matriz Doehlert pôde-se determinar as melhores condições para os fatores estudados de forma mais rápida e com menos experimentos quando comparado com o método de otimização univariada.

O procedimento proposto apresentou RSD\% sempre inferior a 5\% e ótima exatidão, evidenciada pela análise adição e recuperação nas amostras de água, sendo que as recuperações obtidas foram quantitativas na faixa de 94 a 98\%, com baixos limites de detecção e quantificação. Dentre as espécies químicas avalidadas no efeito de matriz, somente o íon cloreto pode interferir na coprecipitação

Tabela 6. Comparação do procedimento proposto com alguns estudos de coprecipitação de cobre e determinação por FAAS

\begin{tabular}{|c|c|c|c|c|c|c|c|}
\hline Agente carreador & Amostra & VA & VD & $\mathrm{FE}$ & $\mathrm{IC}$ & $\mathrm{LD}\left(\mu \mathrm{g} \mathrm{L}^{-1}\right)$ & Referências \\
\hline Hidróxido de túlio & Água e sedimento & 25 & 5 & 50 & 0,5 & 0,51 & 7 \\
\hline Hidróxido de zircônio(IV) & Peixes, água e folhas de chá & 25 & 10 & 25 & 1,0 & 1,55 & 15 \\
\hline Hidróxido de disprósio(II) & Água e sal & 500 & 2 & 250 & 2,0 & 22,0 & 18 \\
\hline $\mathrm{Ni}^{2+} / 2$-nitroso-1naftol-4-ácido sulfônico & Líquen e água do rio & 50 & 2 & 25 & 2,0 & 1,30 & 19 \\
\hline Hidróxido de gadolínio & Água & 10 & 2 & 5 & 2,0 & 3,00 & 24 \\
\hline $\mathrm{Bi}(\mathrm{III}) 4-\mathrm{MPDC}$ & Água do mar, rio e torneira & 1000 & 5 & 200 & 5,0 & 0,50 & 26 \\
\hline Triazol derivatizado & Solo, água e folhas de chá & 25 & 5 & 50 & 0,5 & 0,56 & 30 \\
\hline Hidróxido de érbio & Urina, solo, sedimento e água & 10 & 2 & 25 & 0,4 & 1,10 & 31 \\
\hline Hidróxido de alumínio & Água do mar e água mineral & 50 & 2,5 & 125 & 0,4 & 3,00 & 32 \\
\hline MEFMAT & Água do mar e folhas de chá & 50 & 5 & 50 & 1,0 & 1,49 & 33 \\
\hline APSAL & Água, folha de maçã e chá & 50 & 5 & 100 & 0,5 & 0,50 & 34 \\
\hline QAN & Folhas de chá e pêssego & 25 & 5 & 100 & 0,25 & 0,23 & 45 \\
\hline Cromato de prata & Água mineral e subterrânea & 10 & 0,2 & 50 & 0,2 & 0,23 & Este trabalho \\
\hline
\end{tabular}

VA-volume de amostra (mL); VD-volume do dispersor (mL); FE-fator de enriquecimento; LD-limite de detecção; IC-índice de consumo; MPDC: metilpiperidinoditiocarbamato; MEFMAT: 2-\{[4-(4-fluorofenil)-5-sulfanil-4H-1,2,4-triazol-3-il]metil\}4-\{[(4-fluorofenil)metileno]amino\}-5-(4-metilfenil)-2,4-dihidro3H-1,2,4-triazol-3-ona); APSAL: 4-(2-hidroxibenzilidenoamino)-1,2-dihydro-2,3-dimetil-1-fenilpirazol-5-ona QAN: Ácido Benzóico 2-[(E)-(8-hidróxi2metilquinolin-5-ol)diazinil]. 
do cobre, mostrando que o procedimento não é aplicável a amostras consideradas salobras (salinidade compreendida entre $0,50 \%$ e $30 \%$ ).

As principais vantagens do procedimento desenvolvido foram a sua simplicidade, baixo custo e bons parâmetros analíticos. Estes resultados indicam a viabilidade de aplicação deste procedimento de coprecipitação na separação/pré-concentração de cobre e determinação por FAAS.

\section{REFERÊNCIAS}

1. Brewer, G. J. Em Molecular, Genetic, and Nutritional Aspects of Major and Trace Minerals; Collins, J. F., ed.; Elsevier: Amsterdã, 2017, cap. 7-10.

2. Subramanian, K. S.; Spectrochim. Acta, Part B 1996, 51, 291.

3. Mohammadi, S. Z.; Shamspur, T.; Baghelani, Y. M.; Arab. J. Chem. (2014), DOI: 10.1016/j.arabjc.2014.11.054.

4. Marczenko, Z.; Balcerzak, M.; Separation, Preconcentration and Spectrophotometry in Inorganic Analysis, Elsevier B.V.: Amsterdã, 2000.

5. Bishop, M. L.; Fody, E. P.; Schoeff, L. E. Química Clínica, 5a. ed, Manole: Barueri, 2010.

6. Korn, M. D. G. A.; de Andrade, J. B.; de Jesus, D. S.; Lemos, V. A.; Bandeira, M. L. S. F.; dos Santos, W. N. L.; Bezerra, M. A.; Amorim, F. A. C.; Souza, A. S.; Ferreira, S. L. C.; Talanta 2006, 69, 16.

7. Soylak, M.; Aydin, A.; Food Chem. Toxicol. 2011, 49, 1242.

8. Evans, E. H.; Pisonero, J.; Smith, C. M. M.; Taylor, R. N. T.; J. Anal. At. Spectrom. 2013, 29, 779.

9. Hywel Evans, E.; Pisonero, J.; Smith, C. M. M.; Taylor, R. N.; J. Anal. At. Spectrom. 2015, 30, 1017.

10. Camel, V.; Spectrochim. Acta, Part B 2003, 58, 1177.

11. Bezerra, M. D. A.; Zezzi Arruda, M. A.; Costa Ferreira, S. L.; Appl. Spectrosc. Rev. 2007, 40, 269.

12. Jain, V. K.; Pillai, S. G.; Mandalia, H. C.; TrAC, Trends Anal. Chem. 2016, $85,46$.

13. Alexandrova, L.; Grigorov, L.; Int. J. Miner. Process. 1996, 48, 111.

14. Ilaiyaraja, P.; Singha Deb, A. K.; Sivasubramanian, K.; Ponraju, D.; Venkatraman, B.; J. Hazard. Mater. 2013, 250-251, 155.

15. Citak, D.; Tuzen, M.; Soylak, M.; Food Chem. Toxicol. 2009, 47, 2302.

16. Soylak, M.; Narin, I.; Divrikli, U.; Saracoglu, S.; Elci, L.; Dogan, M.; Anal. Lett. 2004, 37, 767.

17. Alothman, Z. A.; Unsal, Y. E.; Habila, M.; Tuzen, M.; Soylak, M.; Desalin. Water Treat. 2015, 53, 3457.

18. Peker, D. S. K.; Turkoglu, O.; Soylak, M.; J. Hazard. Mater. 2007, 143, 555 .
19. Uluozlu, O. D.; Tuzen, M.; Mendil, D.; Soylak, M.; J. Hazard. Mater. 2010, 176, 1032 .

20. Mendil, D.; Karatas, M.; Tuzen, M.; Food Chem. 2015, 177, 320.

21. Elçi, L.; Şahin, U.; Öztaş, S.; Talanta 1997, 44, 1017.

22. Soylak, M.; Saracoglu, S.; Divrikli, U.; Elci, L.; Talanta 2005, 66, 1098

23. Divrikli, Ü.; Elçi, L.; Anal. Chim. Acta 2002, 452, 231.

24. Soylak, M.; Balgunes, H.; J. Hazard. Mater. 2008, 155, 595.

25. Feist, B.; Mikula, B.; Food Chem. 2014, 147, 225.

26. Efendioğlu, A.; Yağan, M.; Batı, B.; J. Hazard. Mater. 2007, 149, 160

27. Alfasi, Z. B.; Wai, C. M.; Preconcentration Techniques for Trace Elements, CRC Press: Boca Raton, 1992.

28. Zhu, C.; Geochim. Cosmochim. Acta 2004, 68, 3327.

29. Barnes, J. D.; Thomas, M. J. K.; Denney, R. C.; Mendham, J.; Vogel Análise Química Quantitativa, 5a. ed., LTC: Rio de Janeiro, 2002.

30. Bahadır, Z.; Bulut, V. N.; Ozdes, D.; Duran, C.; Bektas, H.; Soylak, M.; J. Ind. Eng. Chem. 2014, 20, 1030.

31. Saracoglu, S.; Soylak, M.; Elci, L.; Talanta 2003, 59, 287.

32. Doner, G.; Ege, A.; Anal. Chim. Acta 2005, 547, 14.

33. Duran, C.; Ozdes, D.; Sahin, D.; Bulut, V. N.; Gundogdu, A.; Soylak, M.; Microchem. J. 2011, 98, 317.

34. Gouda, A. A.; Talanta 2016, 146, 435.

35. Soylak, M.; Onal, G.; J. Hazard. Mater. 2006, 137, 1130.

36. Yıldız, E.; Saçmacı, Ş.; Kartal, Ş.; Saçmacı, M.; Food Chem. 2016, 194, 143.

37. Ferreira, S. L. C.; Dos Santos, W. N. L.; Quintella, C. M.; Neto, B. B.; Bosque-Sendra, J. M.; Talanta 2004, 63, 1061

38. Bezerra, M. A.; Santelli, R. E.; Oliveira, E. P.; Villar, L. S.; Escaleira, L.; Talanta 2008, 75, 965.

39. Lundstedt, T.; Chemom. Intell. Lab. Syst. 1998, 42, 3.

40. Pimentel, M. F.; Barros Neto, B.; Quim. Nova 1996, 19, 268.

41. Novaes, C. G.; Yamaki, R. T.; de Paula, V. F.; do Nascimento Júnior, B. B.; Barreto, J. A.; Valasques, G. S.; Bezerra, M. A.; Rev. Virtual Quim. 2017, 9, 1184.

42. Passari, L. M. G. Z.; Soares, P. K.; Bruns, R. E.; Quim. Nova 2011, 34, 888.

43. Vogel, A. I.; Quimica Analitica Qualitativa, 1a. ed, Mestre Jou: São Paulo, 1981

44. Thompson, M.; Ellison, S. L. R.; Wood, R.; Pure Appl. Chem. 2002, 73, 835.

45. Green, J. M.; Anal. Chem 1996, 68, 305.

46. Rosini, F.; Matos, W. O.; Santos, M. C.; Nóbrega, J.; Analytica 2006, 22,74 . 